\title{
Configurações
}

Revista de sociologia

\section{Saúde mental e utilização de psicofármacos em crianças e jovens em regime de acolhimento residencial em Portugal - um estudo exploratório}

Mental health and Psychotropic Medication Use among Children and Young People in Residential Care - An exploratory study

La santé mentale et l'utilisation des médicaments psychotropes chez les enfants les jeunes placés en institutions - une étude exploratoire

Joana Pires e Paula Cristina Martins

\section{OpenEdition}

Edição electrónica

URL: http://journals.openedition.org/configuracoes/7165

DOI: $10.4000 /$ configuracoes. 7165

ISSN: 2182-7419

Editora

Centro de Investigação em Ciências Sociais

Edição impressa

Paginação: 123-137

ISSN: 1646-5075

\section{Refêrencia eletrónica}

Joana Pires e Paula Cristina Martins, « Saúde mental e utilização de psicofármacos em crianças e jovens em regime de acolhimento residencial em Portugal - um estudo exploratório », Configurações [Online], 23 | 2019, posto online no dia 28 junho 2019, consultado o 30 junho 2019. URL : http:// journals.openedition.org/configuracoes/7165; DOI : 10.4000/configuracoes.7165 
Pires, Joana; Martins, Paula Cristina - Saúde mental e utilização de psicofármacos em crianças e jovens em regime de acolhimento residencial em Portugal - um estudo exploratório. Configurações, vol. 23, 2019, pp.123-137.

\title{
Saúde mental e utilização de psicofármacos em crianças e jovens em regime de acolhimento residencial em Portugal - um estudo exploratório
}

\author{
JOANA PIRES* \\ Universidade do Minho \\ PAULA CRISTINA MARTINS ** \\ Universidade do Minho
}

\section{Resumo}

A elevada prevalência de problemas de comportamento e de perturbações de saúde mental das crianças e jovens nas instituições dos sistemas de proteção são internacionalmente reconhecidas. A medicação psicotrópica constitui uma modalidade de intervenção nestas condições cuja utilização tem vindo a aumentar de forma consistente nos últimos anos, desconhecendo-se todavia as suas implicações a médio e longo prazo no funcionamento e desenvolvimento destas crianças e jovens. Neste artigo, a partir da caraterização dos problemas do foro psicológico e comportamental das crianças e jovens com medidas de acolhimento residencial em 11 instituições portuguesas e das modalidades de intervenção de que são objeto, procura-se discutir a utilização de psicofármacos neste contexto.

Palavras-chave: Saúde mental, psicofármacos, acolhimento residencial.

\footnotetext{
Abstract

Mental health and Psychotropic Medication Use among Children and Young People in Residential Care - An exploratory study

The high prevalence of behavioral and mental health problems among children and youth involved in the child welfare system is widely recognized. Psychotropic medication is a form of intervention in these conditions that has consistently increased in

*E-mail: a71972@alunos.uminho.pt

**E-mail: pcmartins@psi.uminho.pt
} 
recent years, despite the fact that their long term effects on children's functioning and development are not yet fully understood. In this paper, based on the characterization of emotional and behavioral problems of children and youth living in 11 Portuguese residential care homes and of the intervention strategies used to address them, we discuss the current use of psychotropic drugs in this context.

Keywords: Mental health, psychotropic drugs, residential child care.

\section{Résumé}

La santé mentale et l'utilisation des médicaments psychotropes chez les enfants les jeunes placés en institutions - une étude exploratoire

La prévalence élevée des problèmes comportementaux et mentaux chez les enfants et les jeunes accueillis en institutions est largement reconnue. Les médicaments psychotropes sont une modalité d'intervention dans ces conditions, dont leur utilisation n'a cessé d'augmenter au cours des dernières années, bien que ses effets sur le fonctionnement et le développement de ces enfants et adolescents ne sont pas encore totalement connus. Dans cet article, basé sur la caractérisation des problèmes psychologiques et de comportement des enfants et des jeunes accueillis dans 11 institutions du système de protection Portugais et sur les modalités d'intervention qui y sont adoptées, nous discutons l'utilisation actuelle des psychotropes dans ce contexte.

Mots-clés: Santé mentale, médicaments psychotropes, accueil des enfants et des jeunes.

\section{Psicopatologia de crianças e jovens em contexto institucional}

A prevalência de perturbações psiquiátricas diagnosticadas na infância tem vindo a aumentar de forma significativa (Batstra et al., 2012). A nível mundial, a Organização Mundial de Saúde estima que cerca de 20\% das crianças e adolescentes apresente pelo menos uma perturbação mental antes de atingir os 18 anos de idade (World Health Organization, 2000). Já Polanczyk, Salum, Sugaya et al. (2015) referem um valor em torno dos $13.4 \%$ para este grupo populacional. No caso de Portugal, a informação disponível é escassa, sabendo-se, no entanto, que é o país europeu com maior prevalência de doenças mentais na população adulta (Marques, Torrado, Natário e Proença, 2011).

Em particular, na população que vive em contexto de acolhimento residencial, a prevalência de perturbações mentais é mais elevada quando comparada com a população que vive em contextos normativos de vida (McCann, James, Wilson e Dunn, 1996; Scozzaro e Janikowski, 2015). De facto, as crianças e adolescentes que têm medida de acolhimento em instituição registam elevados níveis de problemas comportamentais e emocionais (Desjardins, Lafortune e Cyr, 2017; DosReis, Tai, Goffman, Lynch, Reeves e Shaw, 2014; Scozzaro e Janikowski, 2015), ao que não serão alheias as experiências adversas e traumáticas que marcam os seus percursos de vida (Solchany, 2011), associadas ao stress induzido pela própria condição de acolhimento (Crismon e Argo, 
2009; Solchany, 2011). Num estudo conduzido por Linares, Martinez-Martin e Castellanos (2013) nos EUA, com um grupo de 252 crianças e adolescentes entre os 3 e 14 anos de idade, pouco tempo depois da sua entrada na instituição de acolhimento, verificou-se que $89 \%$ preenchiam os critérios de, pelo menos, uma perturbação mental e $23 \%$ de duas perturbações diferentes, respetivamente. Mais recentemente, numa amostra de 128 crianças norte-americanas em regime de acolhimento, Scozzaro e Janikowski (2015) encontraram 59\% casos com algum tipo de perturbações mental, o que, segundo os autores, foi consistente com os dados de estudos anteriores. Já Bronsard et al. (2016), numa metanálise da prevalência de perturbações mentais das crianças e jovens no sistema de proteção, maioritariamente com medida de colocação, estimaram um valor de $49 \%$. De uma forma geral, na investigação neste domínio, os problemas mais frequentemente identificados foram a perturbação de hiperatividade e défice de atenção (PHDA), perturbação de oposição e desafio, depressão, ansiedade e perturbação de stress pós traumático (PTSD) (Desjardins et al., 2017; DosReis et al., 2014; Green, Hawkins e Hawkins, 2005; Linares, Martinez-Martin e Castellanos, 2013; McAuley e Davis, 2009; Narendorf, Bertram e McMillen, 2011).

Em Portugal, de acordo com o relatório de Caracterização Anual da Situação de Acolhimento das Crianças e Jovens (CASA), em 2016, e à semelhança do que foi identificado em anos anteriores, esta população apresenta uma variedade de problemas de saúde mental e de comportamento ${ }^{1}$ (ISS, 2017). Os problemas de comportamento continuam a ser os mais comuns neste contexto, registando um aumento sistemático de ano para ano. De acordo com o mesmo relatório, em 2016, 27\% do total das crianças e jovens acolhidos foram identificados com esta problemática, mantendo a sua predominância no grupo etário dos 15 aos 17 anos (55\%), seguido do grupo etário dos 12 aos 14 anos (23\%). Das 2227 crianças e jovens nesta situação, em $72 \%$ o comportamento foi considerado do tipo ligeiro, estando relacionado com a perturbação de oposição e em $4 \%$ o comportamento considerou-se do tipo grave, relacionando-se com condutas antissociais (ISS, 2017). Relativamente aos problemas de saúde mental, que não são especificados no relatório, 393 crianças e jovens foram clinicamente diagnosticados, menos 65 do que no ano de 2015 (ISS, 2016; 2017). Esta problemática é identicamente predominante no grupo etário dos 15 aos 17 anos, com 179 jovens identificados, correspondendo assim a uma percentagem de $46 \%$, seguido do grupo etário dos 12 aos 14 anos, com uma percentagem de $21 \%$.

1 Incluem-se aqui as expressões emocionais e comportamentais identificadas pelo entorno das crianças/ jovens como problemas e que podem ou não ser diagnosticadas como perturbações mentais ou ter uma prescrição farmacológica associada. 


\section{Do diagnóstico psicopatológico à intervenção farmacológica}

Associada à presença de psicopatologia está a prescrição e consumo de psicofármacos. Vários estudos indicam a utilização desta prática como forma de responder aos problemas apresentados pelas crianças e adolescentes em regime de acolhimento (Brenner, Southerland, Burns, Wagner e Farmer, 2014; Green, Hawkins e Hawkins, 2005; Linares, Martinez-Martin e Castellanos, 2013; Narendorf, Bertram e McMillen, 2011; Breland-Noble, Elbogen, Farmer, Dubs, Wagner e Burns, 2004; Zito, Safer, Sai, Gardner, Thomas, Coombes e MendezLewis, 2008). Há evidência de que o uso deste tipo de medicamentos é muito frequente neste grupo de crianças e jovens (Zito et al., 2008), sendo duas a três vezes mais elevado comparativamente com a população em geral (Raghavan, Zima, Andersen, Leibowitz, Schuster e Landsverk, 2005). Os diferentes estudos que incidem na prescrição de psicofármacos para este grupo populacional apresentam uma grande amplitude de resultados, entre 12 e $77 \%$ (Brenner et al., 2014; Desjardins, Lafortune e Cyr, 2017; DosReis et al., 2014; Green, Hawkins e Hawkins, 2005; Linares, Martinez-Martin e Castellanos, 2013), podendo esta amplitude de resultados decorrer do tamanho das amostras selecionadas. Em Portugal, de acordo com os relatórios CASA (ISS, 2016; 2017), em 2015, 23,4\% das crianças e adolescentes estavam medicados, tendo essa percentagem diminuído para 20\%, em 2016. De uma forma geral, e de acordo com Mackie et al. (2011), a literatura relevante, incluindo estudos científicos e relatórios oficiais, aponta no sentido de uma utilização excessiva de medicação psicotrópica para gestão de problemas de ordem emocional e comportamental das crianças e jovens em regime de acolhimento, assim como alerta para a utilização simultânea de diferentes tipos de substâncias psicoativas (polifarmacologia) (Breland-Noble et al., 2004; Brenner et al., 2014; Desjardins, Lafortune e Cyr, 2017, 2017; Green, Hawkins e Hawkins, 2005; Narendorf, Bertram e McMillen, 2011). A literatura internacional aponta como psicofármacos mais frequentemente prescritos os psicoestimulantes, os antipsicóticos e os antidepressivos (Desjardins, Lafortune e Cyr, 2017; Green, Hawkins e Hawkins, 2005; Linares, Martinez-Martin e Castellanos, 2013; Narendorf, Bertram e McMillen, 2011; Zito et al., 2008). Em algumas situações, a medicação prescrita é reforçada ao longo do desenvolvimento destas crianças e jovens (DosReis et al., 2014).

A medicação psicofarmacológica não é isenta de riscos e de efeitos colaterais (Crismon e Argo, 2009; Cruz, Lemos, Piani e Brigagão, 2016; Solchany, 2011; Underwood e Washington, 2016). O impacto dos psicofármacos em crianças e adolescentes pode observar-se a curto e longo prazo, tendo influência em diferentes áreas das suas vidas. A sintomatologia secundária e efeitos colaterais de curto prazo dos fármacos é corroborada por um corpo consistente de investigação. Releve-se a este propósito, no grupo dos psicoestimulantes, o metilfenidato, atualmente o fármaco mais utilizado no tratamento 
da Perturbação de Hiperatividade com Défice de Atenção (PHDA), conhecido pelo nome comercial de Ritalina (Cruz et al., 2016; DGS, 2015). Os estudos descrevem como efeitos secundários mais frequentes a apatia, embotamento cognitivo e afetivo, sonolência, insónia, alucinações e problemas cardiovasculares. Numa pesquisa que incide em dez anos de publicações, Itaborahy e Ortega (2013) acrescentam cefaleias, redução do apetite e consequente perda de peso e menor crescimento. Em relação aos efeitos menos frequentes, referem também a dependência, depressão e aumento da ansiedade, entre outros. Para além dos psicoestimulantes, são frequentemente prescritos a estas crianças e jovens antidepressivos, utilizados no tratamento de perturbações de ansiedade, perturbações obsessivo-compulsivas, enurese e também PHDA (Wilens, 2016). Da medicação antidepressiva pode resultar sedação, tonturas, náuseas, insónia, ganho ou perda de peso, nervosismo, ansiedade, tremores e palpitações (Brasil e Belisário Filho, 2000), assim como a redução da capacidade de experienciar emoções (Solchany, 2011). No caso dos antipsicóticos, usados com o objetivo de tratar a agitação motora ou agressividade severa e a labilidade emocional (Wilens, 2016), as reações adversas associadas podem incluir sedação, delírios, irritabilidade, tristeza (Brasil e Belisário Filho, 2000) e, especialmente no caso dos antipsicóticos atípicos, aumento de peso e obesidade infantil (Shin, Bregman, Frazier e Noyes, 2008 citados em Ninan, Stewart, Theall, King, Evans, Baiden e Brown, 2014). Se o consumo prolongado de psicofármacos na infância e adolescência influencia o funcionamento atual e bem-estar das crianças e jovens, com repercussões a médio e longo prazo, já o impacto no seu desenvolvimento, de acordo com o tipo, conjugação e duração da medicação está largamente por determinar (Lòpez-De Fede, Vyavaharkar e Bellinger, 2014).

A prescrição de psicofármacos é uma consequência do diagnóstico de uma perturbação mental. Contudo, este, por si próprio, também não é isento de consequências relacionais e sociais, que a medicação pode acentuar. Alguns psicofármacos podem afetar a autoestima e autodeterminação dos indivíduos em desenvolvimento e interferir na sua capacidade de relacionamento com os outros (Breggin, 2014; Solchany, 2011). De facto, frequentemente influenciam a forma como os adultos e mesmo os pares passam a lidar com estas crianças e jovens (Brzozowski e Caponi, 2013) e também como estas se veem a si próprias e entendem o seu comportamento. Como Conrad (2006) salienta, se por um lado, a perturbação mental, a respetiva medicação e os seus efeitos legitimam a sua incapacidade para regular o seu comportamento, desresponsabilizando-os pelas suas consequências, por outro podem funcionar como um rótulo social, na base de possíveis discriminações destas crianças e jovens.

Não se ignora que a utilização de psicofármacos, em conjugação ou não com outras modalidades de intervenção, pode ser adequada e eficaz no 
tratamento dos problemas de saúde mental apresentados por crianças e adolescentes. Contudo, as preocupações que esta prática levanta estão relacionadas com a generalização do seu uso, com o modo como a medicação é prescrita e adaptada a cada caso e com as suas implicações a longo prazo. Estas questões ganham particular acuidade relativamente a crianças e jovens, cujos sistemas psicofisiológicos, afetivos e cognitivos estão ainda em formação, mais ainda quando se encontram numa situação de especial vulnerabilidade, sob a responsabilidade do Estado, numa condição que dificulta a sua visibilidade social, voz e representação.

O recurso frequente a esta estratégia de intervenção em contexto de acolhimento (Zito et al., 2008) encontra justificação na escassez de respostas apropriadas e na dificuldade de encontrar serviços de apoio aos problemas evidenciados por estas crianças e jovens, particularmente no domínio da saúde mental (Pimentel, Antão e Ramos, 2015; Rodríguez, 2013). A prescrição médica constitui assim um meio de fácil acesso em comparação com outros serviços psicológicos (Graham, 2008; March, 2007), sendo a medicação de fácil manipulação e reconhecida eficácia no controlo imediato do comportamento e no tratamento do sintoma (March, 2007; Rodríguez, 2013). Contudo, intervenções terapêuticas efetivas requerem modalidades de intervenção integradas que, por vezes, até dispensam o uso de psicofármacos (Crismon e Argo, 2009). Salientam-se neste âmbito as estratégias de intervenção psicoterapêuticas e psicoeducativas, indispensáveis no tratamento dos problemas manifestos, na redução de danos e na prevenção de outros associados (Díaz e Bujones, 2014), para além da promoção do desenvolvimento e funcionamento individual.

Dada a importância deste tópico e a escassez de estudos sobre o mesmo em Portugal, no âmbito de uma investigação mais vasta sobre as modalidades de intervenção nos contextos de acolhimento residencial de crianças e jovens em perigo em Portugal, este estudo visou: (a) caraterizar os problemas de saúde mental apresentados por crianças e jovens com medida de proteção de acolhimento residencial, (b) caraterizar as modalidades de intervenção de que estas crianças e jovens são objeto, (c) caraterizar a utilização dos psicofármacos no quadro da intervenção técnica.

Pretende-se assim contribuir para o conhecimento mais diferenciado das necessidades das crianças e jovens em acolhimento residencial, no nosso país e da intervenção nos problemas emocionais e comportamentais destas crianças e jovens.

\section{Método}

\subsection{Participantes}

A amostra foi constituída por 149 crianças e jovens, de idades compreendidas entre 2 e 23 anos, com medida de acolhimento residencial aplicada em 11 Casas de Acolhimento do concelho de Lisboa. A maioria dos participantes 
situava-se na faixa etária dos 12-17 anos $(43.7 \%, \mathrm{n}=65) ; 30.9 \%(\mathrm{n}=46)$ tinha entre 6-11 anos e $12 \%(\mathrm{n}=18)$ tinha 5 anos ou menos. Note-se que $13.4 \%$ $(\mathrm{n}=20)$ dos jovens acolhidos nestas instituições à data do estudo eram adultos. A maioria $(64.4 \%, n=96)$ era do sexo masculino. Relativamente ao período de permanência na instituição, 29.5\% estava em acolhimento residencial há um ano ou menos, $22.8 \%$ entre $1-2$ anos, $28.2 \%$ entre $3-5$ anos e $19.5 \%$ estava há 6 anos ou mais. O motivo predominante da medida de acolhimento foi a negligência parental, em $59.7 \%$ das crianças e jovens ( $n=89)$, seguido da exposição a comportamentos que comprometem o bem-estar e desenvolvimento da criança ou jovem $(n=52 ; 34.9 \%)$ e dos maus tratos psicológicos $(n=42 ; 28.2 \%)$.

\subsection{Medidas}

A partir da revisão da literatura académica da especialidade, da realização de entrevistas semiestruturadas a informantes-chave e da análise documental de processos de crianças e jovens em regime de acolhimento, foi elaborado um questionário que, na sua versão inicial, foi completado e submetido ao método da reflexão falada com o psicólogo de uma instituição, no sentido de verificar a sua compreensibilidade e adequação aos objetivos propostos (Almeida e Freire, 2010). O questionário foi organizado em cinco grupos de questões: (i) Caraterização da instituição; (ii) Identificação de problemas emocionais e comportamentais das crianças e jovens; (iii) Diagnóstico das perturbações mentais e estratégias de intervenção; (iv) Eficácia da intervenção psicofarmacológica e efeitos secundários e (v) Recursos e necessidades da instituição para a intervenção nos problemas emocionais e comportamentais. O preenchimento da versão final do questionário teve a duração aproximada de 50 minutos e pressupôs a consulta dos processos individuais das crianças e jovens das instituições.

\subsection{Procedimento}

Uma vez identificadas as instituições de acolhimento participantes no estudo, foi solicitada a autorização da direção das mesmas para o envio a cada uma do questionário, em suporte online, através do software LimeSurvey Professional, versão 3.7.1, tendo sido previsto um período de 15 dias para o seu preenchimento pelos respetivos técnicos.

O procedimento adotado garantiu a confidencialidade e o anonimato dos intervenientes e a sua utilização para fins estritos de investigação. Além disso, não é possível atribuir dados específicos a crianças ou jovens identificáveis, uma vez que essa informação não foi solicitada. Foram cumpridos os requisitos éticos e deontológicos aplicáveis, nomeadamente a submissão do estudo à Comissão de Ética da Universidade do Minho. 


\subsection{Estratégia de análise de dados}

Para efeitos deste artigo, apresentam-se as análises descritivas (frequências, médias, desvio-padrão e percentagens) que permitem caraterizar: a) a prevalência e os tipos de problemas emocionais e de comportamento manifestados pelas crianças e jovens em acolhimento; b) o tipo de perturbações diagnosticados; c) as modalidades de intervenção desencadeadas nas instituições de acolhimento para lidar com estes problemas/perturbações; d) a prevalência de casos de medicação com psicotrópicos no grupo em estudo; e) os psicofármacos mais frequentemente prescritos; f) a importância atribuída à intervenção psicofarmacológica.

\section{Resultados}

\subsection{Os problemas emocionais e de comportamento das crianças e jovens em acolhimento residencial}

Quando se pergunta aos técnicos das instituições se as crianças e jovens nelas acolhidos apresentam problemas de comportamento e/ou emocionais, $45.4 \%(n=5)$ referem que atualmente todas apresentam este tipo de problemas; $36.4 \%(n=4)$ consideram que é a maioria e 18.2\% ( $n=2)$ entendem que algumas. Note-se que nenhum técnico considera que nenhuma criança/jovem atualmente em acolhimento tem problemas desta ordem. De acordo com 90.9\% ( $n=10)$ dos técnicos, estes problemas já eram patentes à data de entrada na instituição em todas ou, pelo menos, na maioria das crianças e jovens. Todos convergem na afirmação de que, das crianças e jovens acolhidos, algumas (72.7\%), a maioria $(9.1 \%)$ ou todas $(18.2 \%)$ vieram a manifestar (outros) problemas após a entrada na instituição.

Entre estes problemas, a baixa tolerância à frustração $(61,7 \%)$, a impulsividade $(55 \%)$ e as alterações do humor $(47,7 \%)$ são os que afetam mais crianças e jovens nas instituições compreendidas neste estudo. Seguem-se as dificuldades relacionadas com a aprendizagem e o rendimento escolar $(45,6 \%)$ e as dificuldades de concentração (40,9\%). O incumprimento de regras $(35,6 \%)$, as dificuldades de relacionamento interpessoal e de integração social no grupo de pares $(34,9 \%)$ e os comportamentos de oposição/desafio $(32,9 \%)$ são problemas atribuídos a um número de crianças/jovens que, no seu conjunto, oscila entre os 53 e os 49, respetivamente. Relativamente menos expressivos são nesta amostra os problemas relacionados com o consumo de bebidas alcoólicas $(1.3 \%)$, o vandalismo $(3,4 \%)$, o consumo de estupefacientes $(5,4 \%)$ ou as fugas $(6 \%)$.

Nem todas as crianças/jovens que têm problemas do foro emocional e/ ou comportamental têm diagnóstico de perturbação mental. De facto, $24.2 \%$ $(n=36)$ com este tipo de problemas ou dificuldades não têm diagnóstico psiquiátrico. Das 149 crianças/jovens acolhidos, 33.6\% (n=50) estão diagnosticados com pelo menos uma perturbação mental. Destes 50, 68\% (n=34) estão 
diagnosticados com uma perturbação mental, 24\% ( $\mathrm{n}=12)$ com duas perturbações mentais e $8 \%(n=4)$ com mais do que duas perturbações mentais.

Relativamente às perturbações mentais (Figura 1), aquela que mais frequentemente é diagnosticada nestas crianças/jovens é a perturbação de hiperatividade e défice de atenção, verificada em $40 \%$, seguida da perturbação do comportamento $(28 \%)$ e da perturbação de oposição e desafio (16\%). Com expressão idêntica (12\%) seguem-se as perturbações depressiva e de ansiedade, assim como outras perturbações indicadas pelos profissionais, entre as quais se contam a perturbação da personalidade, a perturbação do espectro do autismo, perturbação do vínculo, perturbação do controlo dos impulsos e perturbação do comportamento sexual

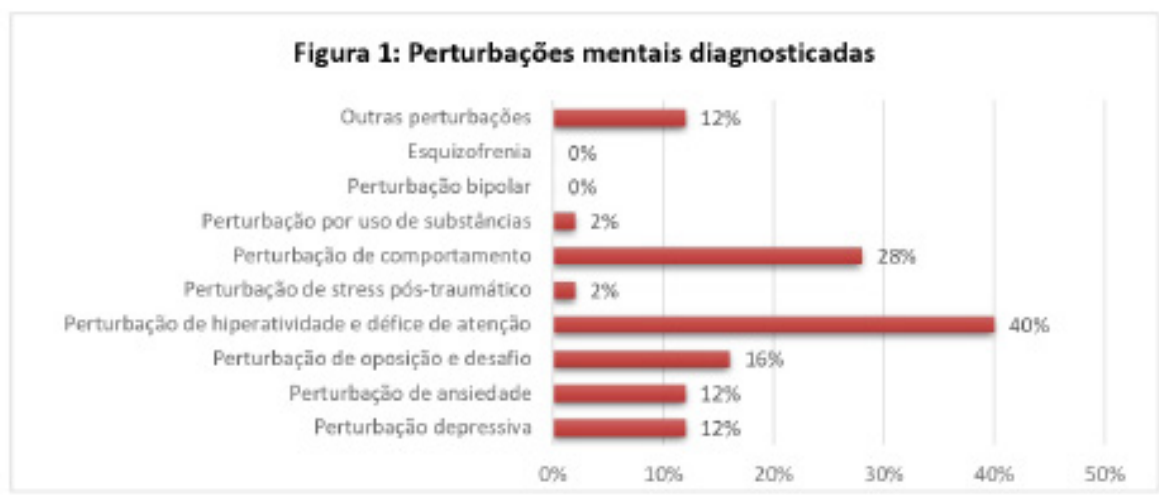

\subsection{Modalidades de intervenção junto das crianças e jovens com pro- blemas de saúde mental em contexto residencial}

O grau de utilização das estratégias de intervenção nas instituições foi avaliado segundo uma escala Likert entre 1 e 5 [1= para nenhuma criança/jovem -5 = para todas as crianças/jovens]. Os resultados (Tabela 1) mostram que as estratégias de intervenção mais frequentemente utilizadas em contexto residencial para as crianças e jovens que apresentam problemas de saúde mental são o acompanhamento individualizado na instituição $(\mathrm{M}=4.82 ; \mathrm{DP}=.04)$, seguido de consulta de medicina geral e familiar $(\mathrm{M}=4.27$; $\mathrm{DP}=1.42)$ e a intervenção familiar $(\mathrm{M}=3.45 ; \mathrm{DP}=1.13)$. 
Tabela 1.

Estratégias de intervenção utilizadas nas instituiçōes de acolhimento residencial

\begin{tabular}{lc}
\hline \multicolumn{1}{c}{ Estratégias de intervençåo } & Grau de utilização \\
\hline Acompanhamento individualizado na instituiçăo & 4,82 \\
\hline Consulta de medicina geral e familiar & 4,27 \\
\hline Intervenção familiar & 3,45 \\
\hline Consulta psicológica/psicoterapia fora da instituição & 3,27 \\
\hline Intervenção escolar especial & 3,18 \\
\hline Envolvimento em programas de grupo na instituição & 2,82 \\
\hline Consulta de pedopsiquiatria & 2,73 \\
\hline Intervenção psicofarmacológica & 2,55 \\
\hline Consulta psicológica na instituição & 1,82 \\
\hline
\end{tabular}

Note-se que a intervenção psicofarmacológica é das modalidades de intervenção menos frequentes. De facto, quando se explora este dado, verifica-se que, no conjunto das 11 instituições inquiridas, à data do estudo, eram medicadas 41 crianças/jovens, o que significa $27.5 \%$ da população total destas casas de acolhimento. Destas, 38 (92.7\%) possuem diagnóstico de perturbação mental. Do total de 41 medicados, a $17.1 \%(\mathrm{n}=7)$ foi-lhes prescrito um psicofármaco, $53.7 \%(\mathrm{n}=22)$ dois psicofármacos e a $29.3 \%(\mathrm{n}=12)$ mais do que dois psicofármacos.

Os grupos de psicofármacos predominantemente utilizados por estas crianças e jovens são os antipsicóticos, os estabilizadores de humor e os psicoestimulantes. Para além da intervenção psicofarmacológica, as crianças e jovens beneficiam de outras estratégias de intervenção. Do total das 41 crianças e jovens medicados, em $68.3 \%(\mathrm{n}=28)$ dos casos está a ser desenvolvido algum tipo de intervenção com as suas famílias, $61 \%(n=25)$ têm acompanhamento especializado na escola, $58.5 \%(\mathrm{n}=24)$ têm acompanhamento psicológico/psicoterapia e $19.5 \%$ ( $n=8)$ estão envolvidos em programas de grupo dentro da instituição.

No que diz respeito à importância atribuída pelos 11 técnicos à intervenção psicofarmacológica, apenas três consideram que esta estratégia é a mais eficaz entre todos os outros tipos de intervenção, sendo para um dos profissionais que respondeu ao questionário uma modalidade de intervenção suficiente. Todos ( $\mathrm{n}=11$ ) a consideram necessária e, para oito técnicos, dificilmente outra 
forma de intervenção é eficaz se não for combinada com a administração de psicofármacos.

\section{Discussão}

Este estudo permitiu confirmar a elevada prevalência de problemas emocionais e de comportamento na população em acolhimento residencial, na sua maior parte já evidentes quando as crianças e jovens dão entrada no sistema, tendendo a observar-se a sua problematização crescente ao longo da duração da medida. Estamos, portanto, perante uma população especialmente vulnerável, o que em grande parte é explicável pelas condições psicossociais e de maus-tratos que estão na origem do seu acolhimento, também verificadas por este grupo de estudo, e que constituem fatores de risco para o desenvolvimento de perturbações de saúde mental (Jozefiak, Kayed, Rimehaug, Wormdal, Brubakk e Wichstrøm, 2016). Salientam-se neste quadro as dificuldades inerentes à regulação do comportamento (impulsividade, tolerância à frustração), ao comportamento social (comportamentos de oposição/desafio, cumprimento de regras, integração no grupo de pares) e à aprendizagem e escolaridade, convergentes com as encontradas na literatura da especialidade (Leloux-Opmeer, Kuiper, Swaab e Scholte, 2016). Note-se que condutas de tipo delinquente, consumos de álcool ou estupefacientes, vandalismo e fugas, não têm expressão relevante neste grupo. Estes dados são consistentes com os do relatório CASA (ISS, 2017), que identifica como predominantes os problemas de comportamento ditos de tipo ligeiro.

No que se refere à prevalência de casos de perturbações de saúde mental diagnosticadas, embora a variabilidade dos dados de comparação seja grande, o diagnóstico de uma perturbação mental em $33.6 \%$ da amostra é consideravelmente inferior aos $89 \%$ do estudo de Linares, Martinez-Martin e Castellanos (2013), aos 59\% de Scozzaro e Janikowski (2015) e aos 49\% de Bronsard et al. (2016). Na origem destas diferenças podem estar as caraterísticas das amostras, os métodos de mensuração das perturbações de saúde mental, o funcionamento dos sistemas de proteção e os critérios de elegibilidade para a medida de acolhimento residencial, assim como a cobertura dos serviços de pedopsiquiatria e a articulação dos serviços de proteção com os serviços de saúde mental, eventualmente distinta entre os países.

Os tipos de perturbações identificados são genericamente convergentes com os de outros estudos (Leloux-Opmeer, Kuiper, Swaab e Scholte, 2016), salientando-se a Perturbação de Hiperatividade com Défice de Atenção (PHDA), as perturbações do humor, as perturbações do comportamento e de oposição e desafio. Também a este propósito, os resultados do relatório CASA (2017) são corroborados.

A intervenção técnica junto destas crianças e jovens consiste globalmente no acompanhamento individualizado da criança/jovem na instituição, no 
acompanhamento médico e na intervenção junto da família, sendo a intervenção psicofarmacológica uma das modalidades menos frequentes.

Note-se que a intervenção psicofarmacológica é uma estratégia de intervenção que abrange algo mais de um quarto das crianças e jovens em acolhimento nas instituições inquiridas $(27.5 \%)$, não se tratando, pois, de um recurso frequente ou extensivo. Trata-se de um valor dentro do âmbito amplo de valores obtidos noutros estudos (entre os 12\%-77\%), ainda assim superior aos reportados nos relatórios CASA 2015 e 2016 (ISS, 2016; 2017), respetivamente 23,4\% e $20 \%$. Contudo, mais de metade destes jovens consome simultaneamente dois tipos diferentes de psicofármacos, um exemplo de polifarmacologia. Os psicofármacos mais utilizados são os antipsicóticos, os psicoestimulantes e os estabilizadores do humor, conforme as práticas de outros países (Desjardins, Lafortune e Cyr, 2017; Linares, Martinez-Martin e Castellanos, 2013).

Nos casos de crianças/jovens medicados, a intervenção diferencia-se e especializa-se: além da intervenção familiar, o acompanhamento psicológico/psicoterapêutico, a educação especial e os programas de grupo conduzidos dentro da instituição ganham maior relevância. Este dado parece ir ao encontro da combinação e, eventualmente, da integração de modalidades de intervenção psicoterapêutica, socioeducativa e psicofarmacológica, cuja eficácia é considerada superior à de estratégias de intervenção monomodais. É neste sentido que a maioria dos técnicos entende que a intervenção psicofarmacológica não só é necessária, como também é condição de eficácia de outras formas de intervenção, o que recomenda a sua administração combinada.

Os dados obtidos não substanciam a preocupação inicialmente enunciada com a vulgarização, intensificação e relativa indiferenciação da prática de prescrição e administração de fármacos para controlo do mal-estar e dos comportamentos problemáticos de crianças e jovens no sistema de acolhimento residencial. O facto de estas casas de acolhimento pertencerem a uma só instituição, estando funcionalmente sujeitas à mesma direção, eventualmente adotando as mesmas orientações estratégicas e filosofia de funcionamento, o tamanho limitado da amostra, o reduzido número de diagnósticos de saúde mental das crianças e jovens em acolhimento nestas instituições, poderão contribuir para a explicação destes valores relativamente mais baixos do que os dos estudos realizados neste domínio. Por este motivo, impõe-se o alargamento da amostra a outras instituições, geograficamente dispersas e com hierarquias diferenciadas. 


\section{Referências bibliográficas}

ALMEIDA, L. \& FREIRE, T. (2010), Metodologias da investigação em psicologia e educação (5 $5^{\text {ed. }}$, Braga, Psiquilíbrios.

BATSTRA, L.; HADDERS ALGRA, M.; NIEWEG, E.; VAN TOL, D.; PIJL, S. \& FRANCES, A. (2012), Childhood emotional and behavioral problems: reducing overdiagnosis without risking undertreatment. Developmental Medicine \& Child Neurology, 54(6), 492-494. doi: 10.1111/j.1469-8749.2011.04176.x

BRASIL, H., \& BELISÁRIO FILHO, J. (2000), Psicofarmacoterapia. Revista Brasileira de Psiquiatria, 22, 42-47. doi: 10.1590/S1516-44462000000600012.

BRELAND-NOBLE, A.; ELBOGEN, E.; FARMER, E.; DUBS, M.; WAGNER, H. \& BURNS, B. (2004), Use of Psychotropic Medications by Youths in Therapeutic Foster Care and Group Homes, Psychiatric Services, 55(6), 706-708.

BRENNER, S.; SOUTHERLAND, D.; BURNS, B.; WAGNER, H., \& FARMER, E. (2014), Use of Studies, 23(4), 666-674.

BRONSARD, G ; ALESSANDRINI, M. ; FOND, G. ; LOUNDOU, A. ; AUQUIER, P. ; TORDJMAN, S. \& BOYER, L. (2016), The Prevalence of Mental Disorders among Children and Adolescents in the Child Welfare System. A Systematic Review and MetaAnalysis, Medicine, 95(7), 1-17.

BRZOZOWSKI, F. \& CAPONI, S. (2013), Medicalização dos Desvios de Comportamento na Infância: Aspectos Positivos e Negativos, Psicologia: Ciência e Profissão, 33(1), 208-221.

BUJES, J. (2014), Tecnologias de Governo, Práticas de Constituição de Sujeitos e Subjetividades no atendimento de adolescentes infratores internados na FASE/RS, Anais da ReACT, vol. 1, $\mathrm{n}^{\circ}$ 1. Comunicação apresentada na Reunião de Antropologia da Ciência e Tecnologia. Disponível em http://ocs.ige.unicamp.br/ojs/react/article/view/1206/659.

COHEN, E.; PFEIFER, J. \& WALLACE, N. (2014), Use of psychiatric medications in juvenile detention facilities and the impact of state placement policy, Journal of Child and Family Studies, 23, 738-744. doi: 10.1007/s10826-012-9655-4.

CRISMON, M. \& ARGO, T. (2009), The use of psychotropic medication for children in foster care, Child Welfare, $88(1), 71$.

CRUZ, B. ; LEMOS, F.; PIANI, P. \& BRIGAGÃO, J. (2016), Uma crítica à produção do TDAH e a administração de drogas para crianças, Estudos de Psicologia, 21(3), 282-292. doi:10.5935/1678-4669.20160027

DESJARDINS, J. ; LAFORTUNE, D. \& CYR, F. (2017), Psychopharmacotherapy in children placed in group homes and residential centres in Canada: Psychopathological portrait of children receiving psychotropic medications and educators' perception of treatment, Children and Youth Services Review, 81, 197-206.

DÍAZ, H. \& BUJONES, G. (2014), Pedagogia y medidas psicoeducativas para la integración social: estúdio de un caso, Revista de Ciências da Educação, 30(1), 19-33.

DIREÇÃO-GERAL DA SAÚDE (2015), Saúde Mental em Números - 2014, Lisboa, DGS. Disponível em https:/www.dgs.pt/estatisticas-de-saude/estatisticas-de-saude/publicacoes/ portugal-saude-mental-em-numeros-2014.aspx.

DOSREIS, S.; TAI, M.,GOFFMAN, D.; LYNCH, S.; REEVES, G. \& SHAW, T. (2014), Agerelated trends in psychotropic medication use among very young children in foster care, Psychiatric Services, 65(12), 1452-1457.

DUPPONG-HURLEY K.; TROUT A.; CHMELKA, M.; BURNS B.; EPSTEIN, M.; THOMPSON, R. \& DALY, D. (2009), The changing mental health needs of youth admitted to residential group home care, Journal of Emotional and Behavioral Disorders, 17, 164-176 
ESTRADAS, N. (2014), A eficácia e segurança dos psicofármacos em crianças e adolescentes (Tese de Doutoramento). Disponível em http://hdl.handle.net/10451/24414.

GRAHAM, L. (2008), Drugs, labels and (p) ill-fitting boxes: ADHD and children who are hard to teach, Discourse: studies in the cultural politics of education, 29(1), 85-106. doi: 10.1080/01596300701801377.

GREEN, D.; Hawkins, W. \& Hawkins, M. (2005), Medication of children and youth in foster care, Journal of Social Work in Disability \& Rehabilitation, 4(1-2), 43-55.

ILLICH, I. (1975), Medical nemesis, New York, Bantam Books.

INSTITUTO DA SEGURANÇA SOCIAL (2016), CASA 2015 - Relatório de Caracterização Anual da Situação de Acolhimento das Crianças e Jovens, Lisboa, ISS.

INSTITUTO DA SEGURANÇA SOCIAL (2017), CASA 2016 - Relatório de Caracterização Anual da Situação de Acolhimento das Crianças e Jovens, Lisboa, ISS.

ITABORAHY, C. \& ORTEGA, F. (2013), O metifenidato no Brasil: uma década de publicações, Ciência \& Saúde Coletiva, 18(3), 803-816. doi: https://dx.doi.org/10.1590/ S1413-81232013000300026.

JOZEFIAK, T.; KAYED, N.; RIMEHAUG, T.; WORMDAL, A.; BRUBAKK, A. \& WICHSTRØM, L. (2016), Prevalence and comorbidity of mental disorders among adolescents living in residential youth care, European Child and Adolescent Psychiatry, 25, 33-47. DOI 10.1007/s00787-015-0700-x.

LELOUX-OPMEER, H.; KUIPER, C.; SWAAB, H. \& SCHOLTE, E. (2016), Characteristics of Children in Foster Care, Family-Style Group Care, and Residential Care: A Scoping Review, Journal of Child and Family Studies, 25, 2357-2371. doi: 10.1007/s10826-016-0418-5

LINARES, L.; MARTINEZ-MARTIN, N. \& CASTELLANOS, F. (2013), Stimulant and Atypical Antipsychotic Medications for Children Placed in Foster Homes, PLoS ONE 8(1): e54152. https://doi.org/10.1371/journal.pone.0054152.

LÒPEZ-DE FEDE, A.; VYAVAHARKAR, M. \& BELLINGER, J. (2014). Antipsychotic Prescriptions for Children Aged 5 Years or Younger. Do We Need Policy Oversight Standards?, SAGE Open, 1-7. doi: 10.1177/2158244014555116.,

LYONS, C.; WASSERMAN, G.; OLFSON, M.; MCREYNOLDS, L.; MUSABEGOVIC, H. \& KEATING, J. (2013), Psychotropic Medication Patterns among Youth in Juvenile Justice, Administration \& Policy in Mental Health \& Mental Health Services Research, 40(2), 58-68. doi: 10.1007/s10488-011-0378-4.

MACKIE, T.; HYDE, J.; RODDAY, A.; DAWSON, E.; LAKSHMIKANTHAN, R.; BELLONCI, C.; SCHOONOVER, D. \& LESLIE, L. (2011), Psychotropic medication oversight for youth in foster care: a national perspective on state child welfare policy and practice guidelines, Children and Youth Services Review, 22(33), 2213-2220.

MARCH, R. (2007), Claves para la intervención con menores acogidos en recursos residenciales, que presentan conductas problemáticas, Intervención Psicosocial, 16(2), 213-227. Recuperado en 04 de junio de 2018, de http://scielo.isciii.es/scielo. php?script=sci_arttext $\&$ pid=S1132-05592007000200006\&lng=es\&tlng=es.

MARQUES, C.; TORRADO, M.; NATÁRIO, A. \& PROENÇA, M. (2011), Rede de referenciação hospitalar de psiquiatria da infância e da adolescência, Lisboa, Coordenação Nacional para a Saúde Mental.

MCAULEY, C. \& DAVIS, T. (2009), Emotional well-being and mental health of looked after children in England, Child \& Family Social Work, 14(2), 147-155.

MCCANN, J.; JAMES, A.; WILSON, S. \& DUNN, G. (1996), Prevalence of psychiatric disorders in young people in the care system, BMJ, 313(7071), 1529-1530.

MELTZER, H.; GATWARD, R.; CORBIN, T.; GOODMAN, R. \& FORD, T. (2003), The mental health of young people looked after by local authorities in England, London, The Stationery Office. 
MELTZER, H.; LADER, D.; CORBIN, T.; GOODMAN, R. \& FORD, T. (2004), The Mental Health of Young People Looked After by Local Authorities in Scotland-Summary Report, London: Office for National Statistics, London, The Stationary Office.

NARENDORF, S.; BERTRAM, J. \& LONDON, C. (2011), Diagnosis and medication overload? A nurse review of the psychiatric histories of older youth in treatment foster care, Child Welfare, 90(3), 27.

NINAN, A.; STEWART, S.; THEALL, L.; KING, G.; EVANS, R.; BAIDEN, P. \& BROWN, A. (2014), Psychotropic medication monitoring checklists: Use and utility for children in residential care, Journal of the Canadian Academy of Child and Adolescent Psychiatry, 23(1), 38.

PIMENTEL, A.; ANTÃO, J. \& RAMOS, A. (2015), Avaliação de um programa de promoção da saúde mental com crianças institucionalizadas, Psicologia em Revista, 21(2), 218-234. doi: 10.5752/P.1678-9523.2015v21n2p218.

POLANCZYK, G.; SALUM, G.; SUGAYA, L. et al. (2015), Annual research review: a meta-analysis of the worldwide prevalence of mental disorders in children and adolescents, Journal of Child Psychology and Psychiatry, 56, 345-365.

RAGHAVAN, R.; ZIMA, B.; ANDERSEN, R.; LEIBOWITZ, A.; SCHUSTER, M. \& LANDSVERK, J. (2005), Psychotropic medication use in a national probability sample of children in the child welfare system, Journal of Child \& Adolescent Psychopharmacology, 15(1), 97-106.

RIJO, D.; BRAZÃO, N.; BARROSO, R.; SILVA, D.; VAGOS, P.; VIEIRA, A. \& MACEDO, A. (2016), Mental health problems in male young offenders in custodial versus community based-programs: implications for juvenile justice interventions, Child and Adolescent Psychiatry and Mental Health, 10(40), 1-12.

RODRÍGUEZ, A. (2013), Recursos Residenciales para Menores Seriamente Disruptivos: Aportaciones Técnicas a un Debate Social e Institucional, Papeles del Psicólogo, 34(1), 23-31

SCOZZARO, C. \& JANIKOWSKI, T. (2015), Mental health diagnosis, medication, treatment and placement milieu of children in foster care, Journal of Child and Family Studies, 24(9), 2560-2567.

SHELTON, D. (2004), Experiences of detained young offenders in need of mental health care, Journal of nursing scholarship, 36(2), 129-133.

SOLCHANY, J. (2011), "Psychotropic medication and children in foster care: Tips for advocates and judges". Disponível em https://www.americanbar.org/content/dam/aba/administrative/ child_law/PsychMed.authcheckdam.pdf

TEPLIN, L.; ABRAM, K.; MCCLELLAND, G. \& DULCAN, M. (2003), Comorbid Psychiatric Disorders in Youth in Juvenile Detention, Arch Gen Psychiatry, 60(11), 1097-1108. doi:10.1001/archpsyc.60.11.1097.

UNDERWOOD, L. \& WASHINGTON, A. (2016), Mental illness and juvenile offenders, International journal of environmental research and public health, 13(2), 228.

WHITE, S.; VAN TIEGHEM, M.; BRISLIN, S.; SYPHER, I.; SINCLAIR, S.; PINE, D. \& BLAIR, R. (2016), Neural correlates of the propensity for retaliatory behavior in youths with disruptive behavior disorders, American Journal of Psychiatry, 173(3), 282-290. DOI: 10.1176/appi.ajp.2015.15020250.

WILENS, T. (2016), Straight Talk about Psychiatric Medications for Kids (4 ed.), New York, The Guildford Press.

ZITO, J.; SAFER, D.; SAI, D.; GARDNER, J.; THOMAS, D.; COOMBES, P. \& MENDEZLEWIS, M. (2008), Psychotropic medication patterns among youth in foster care, Pediatrics, 121(1), e157-e163. 